\title{
FUTURE OF TIMING ANALYSIS IN VLSI CIRCUITS
}

\author{
Soujanya Avadhani M D \\ Student, Department of Electronics and Communication Engineering, \\ R V College of Engineering, Bangalore, India
}

Kiran V

Associate Professor, Department of Electronics and Communication Engineering, R V College of Engineering, Bangalore, India

\begin{abstract}
As the VLSI technology scales down into the nanometer domain, the on-chip variations have become more unpredictable. They require a more detailed modelling and analysis of Process, Voltage and Temperature corners of the design. These variations which did not have a pronounced effect on larger chips, have now become inevitably significant and cannot be ignored. This paper presents an overview of the existing Timing Analysis methods and identifies their drawbacks. The need for an integrated Timing Analysis tool which has features to model all the on-chip variations is discussed. By employing a PVT aware Timing Analysis tool, the verification engineer can achieve faster timing closure while not compromising on the quality of results.
\end{abstract}

Key words: Timing Analysis, PVT variations, Power Supply noise, Cell delay, Interconnect Delay, Dynamic Voltage Drop, STA Tools.

Cite this Article: Soujanya Avadhani M D and Kiran V, Future of Timing Analysis in VLSI Circuits, International Journal of Electrical Engineering and Technology, 11(4), 2020, pp. 66-71.

https://iaeme.com/Home/issue/IJEET?Volume=11\&Issue $=4$

\section{INTRODUCTION}

Advancements in Electronic Design Automation (EDA) tools are accompanied with boosted increase in complexity of VSLI designs and rapid decrease in the size of VLSI circuits. This has increased the problems in the design of circuits, modelling its parameters, analyzing them and finally implementing the design on Silicon wafer. With an exponential scale-down of feature sizes, unexpected timing failures are being observed on the fabricated chips. These failures have been traced back to improper modelling of Process, Voltage and Temperature variations across a chip which have in turn led to inaccurate estimations in cell delays and interconnect delays. Therefore, it is important to address there critical issues in order to achieve timing closure and subsequently prevent costly on-chip Silicon failures. 


\section{TIMING ANALYSIS IN VLSI CIRCUITS}

In digital VLSI circuits, it is important to determine an upper limit on the delay of all paths from the primary inputs to the primary outputs. Statistical Timing Analysis (STA) is one of the conventional simulation approaches to characterize the timing performance of digital circuits, to determine the critical paths, and to obtain the delay information. It is an exhaustive method of analyzing, debugging and validating the timing performance of a design. A typical STA tool breaks down the complete design into timing paths, calculates the delay on each path by estimating the inter-cell and intra-cell delays and then checks for violations of timing constraints on each path.

\subsection{Timing Paths in a Design}

Each timing path in a design is defined by a unique pair of start point and endpoint. A timing path can be a data path, clock path, clock-gated path or an asynchronous path. A valid timing path can be one of the four types shown in Fig.1 - primary input to register path, register to register path, register to primary output path or input to output path. The valid starting points of a timing path must be either flipflop clock pins or primary inputs and valid end points of a timing path can be flipflop data pins, gated clocks' control pins or primary output ports.
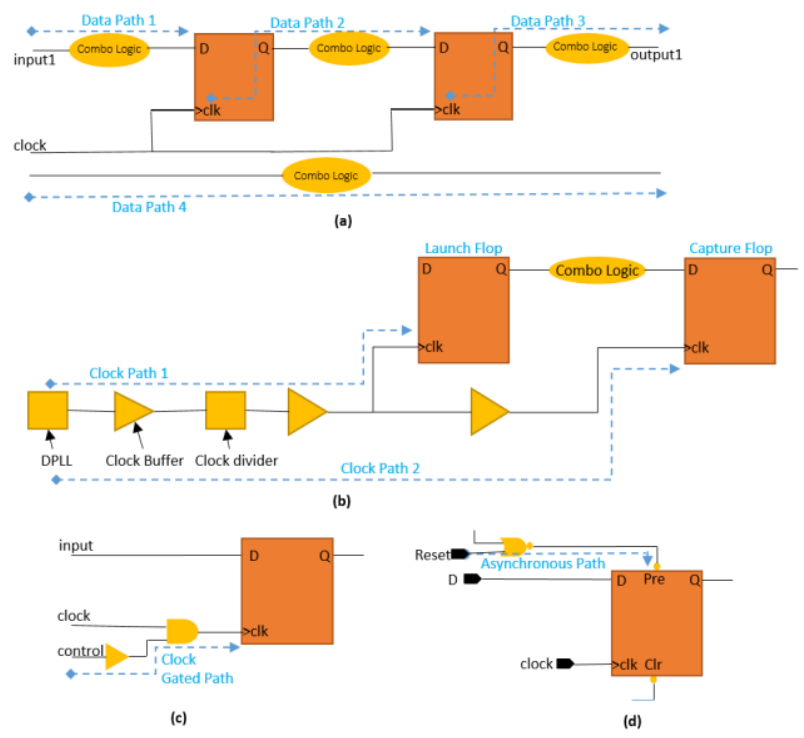

Figure 1 Valid Paths in a design for STA

\subsection{Calculating Path Delay}

Path delay constitutes intra-cell delay, simply known as the cell delay and inter-cell delay which is more commonly known as net delay. STA tools represent netlist as a Directed Acyclic Graph with cells as its nodes and nets as the edges which are correlated with corresponding delay values. Cell delay corresponds to the arc between cell's input and output pins. It is a function of input pin slew and total output load capacitance of each cell. STA tools look up the NLDM table in the liberty file to obtain the cell delay values. Net delay is a function of $R_{n e t}$ and $C_{n e t}$. STA tools obtain net delay values using Wire Load Models that have statistical estimates of $R_{n e t}$ and $C_{n e t}$.

\subsection{Satisfying Path Constraints}

Traditional STA tools check for Setup and Hold time agreements on all the timing paths of the design. The Slack value of each path is dependent on data arrival time, data required time, 
launch and capture flop specifications, input clock skew and clock uncertainty. For a designed circuit to operate as intended, the data should arrive at the capture flop $T_{\text {setup }}$ time units before the capturing clock edge and should not vary at least until $T_{\text {hold }}$ time units after the clock edge. This caps the maximum clock frequency at which the circuit can operate. The STA tools also performs fanout checks, max-cap check, transition checks and input/output delay checks.

\subsection{Problems in Traditional Timing signoff Methodologies}

The Timing Signoff methods implemented in traditional STA tools include the variations in voltage corner, but typically it considers only the cases where all the supplied voltages are either Low or High. This will not capture the actual worst delay on a timing path as it will neglect the possible degradation of supply voltage between successive instances on a path [1]. With the Supply voltages being scaled down and corresponding reduction in device threshold voltage, the circuits are more susceptible to power-bus voltage variations [2]. When there is logical state switching in a circuit, the current flows from VDD pin to Ground pin of a cell. This causes voltage variations in the P-G rails due to the non-ideal nature of the buses in the form of a voltage-drop at the power bus contact and/or a voltage surge at the ground bus contact of the logic gate to the power distribution network. This has an adverse effect on the performance and reliability of the circuit. With increasing clock frequency, the time period and timing margins of a design are reduced. Therefore, it is now absolutely necessary to model these uncertainties while performing a timing signoff.

\section{RECENT DEVELOPMENTS IN TIMING ANALYSIS TECHNIQUES}

In the recent years, many methodologies have been proposed to overcome the inherent drawbacks in traditional STA tools and the Timing Analysis Methodologies they use. They include modelling the effect of Power Supply Noise variations [2-5], modelling cell delays by considering the effect of Dynamic Voltage Drops [1,6] and modelling the thermal effect on path delay [4]. Various new approaches such as path-based simulations for crosstalk analysis and simultaneous analysis of multiple paths [7,8], effective identification of Timing Windows [8], considering derate factors [4] due to On-Chip Variations (OCV) and delay based circuit pruning techniques [6] have been proposed.

\subsection{Modelling of Power Supply Noise}

In [2], the authors suggest estimating the maximum voltage drop at the node by formulating it as an optimization problem. The maximum voltage drop that occurs at a node during a specific time sub-interval (determined by the overlap of uncertainty intervals of the gates) is formulated as an optimization problem considering all the functional inputs of the design. Upon solving the optimization problem, the upper bound on the voltage drop in that subinterval is obtained. In [3], the supply voltage of a logic block is modelled as a random variable and its probability density function is derived from the transistor level simulations on a subset of vectors that trigger high power supply noise. From these statistical models of $P / G$ voltage levels, the perturbation of the probability density function of net delays and cells delays are derived and STA is performed. A non-linear timing analysis technique that does not require additional characterization of standard cell libraries is proposed in [4]. This technique handles the different temperature and supply voltage variations at the cell level. In [5], the power supply noise effects are considered in STA based on the selective cell library characterization and remapping to specific supply voltage values. They propose a method to perform power network analysis and obtain the IR drop value for each cell. An SDF file is generated from the arc delay values calculated from the IR drop values. This SDF file is given as an input to perform STA and re-compute the path delays. 


\subsection{Modelling Cell Delay}

Gate/Cell delay is calculated as a function of input signal slope and output load traditionally. In [1], the authors calculate cell delay as a function of 4 additional parameters which are input high signal level, input low signal level, the gate's power supply and ground levels. A quadratic polynomial is used to represent the dependence of delay on each of these voltage values and an allowance is given for the cross product terms. In [6], the gate delay is modelled as a non-linear function of local supply voltages of the gate itself and supply voltages at the preceding driver gate in addition to input slew and output load capacitances. The authors of [6] opine that practically as voltage drop and ground rise in a circuit can be restricted to $10 \%$ of their nominal values, the delay and output slew of the gate can be approximated as quadratic functions of supply voltage of that gate and its driver.

\subsection{Modelling the Thermal Effect}

Modelling the effect of temperature on the output transition time of a cell is complex in slow input range [4]. It is identified that for a given $V_{D D}$ value, the thermal sensitivity is a decreasing function of temperature and the rate of decrease is significant when the $V_{D D}$ is very small. The sensitivity is almost constant in the fast rising input. For a slow rising input signal, the rise duration defines the waveform of the associated delays.

\subsection{Path Based Approaches for Timing Analysis}

Analyzing the crosstalk delays is critical for a Timing signoff. In traditional STA tools crosstalk delay analysis is performed at a net level which inherently introduces a lot of pessimism when the full path is considered. In STA, the switching time of the victim instance is bound by the earliest and latest arrival times. To compute worst crosstalk delay on a net, the complete switching window of the victim should be considered to ensure that the crosstalk delay computed is the worst for any path through that victim net. In [8], the authors have proposed to analyse the crosstalk delays from a path perspective based on the fact that it discretizes the victim instance switching time which will become less pessimistic. They suggest using a 2 pass approach; in the first pass a superset of all crosstalk delay path violations are identified using net based approach and in the second pass, the raw crosstalk delay setup/hold violations obtained from STA are now re-evaluated. Since the exact failing path is known, the arrival times on the victim nets along the path are known. Since the arrival time on the victim is known, the aggressors to the victim on the path can be picked up more accurately. The crosstalk delay on the victim nets along the failing path is recalculated using the newly picked aggressors. Based on the new crosstalk delay calculated, the slack on the path is re-evaluated. Path based analysis reduces pessimism by using discrete path arrival victim switching, using path constraining clock and path level asynchronous grouping.

Multiple paths can be simultaneously analysed in a single session if each cell has a single voltage annotation for all paths passing through that cell [7]. In order to do this, we identify all the timing windows at the output of every cell, at all clock domain and edges. The minimum of the voltages is taken as the $\mathrm{V}_{\min }$ for STA.

\subsection{Tweaking the Timing Windows of a Cell}

In designs with multiple clock domains, large timing windows for few cells introduces pessimism. In [8], the most constraining timing conditions for setup and hold analysis are identified. To capture a realistic $\mathrm{V}_{\min }$, a small window of 1 cell delay around $\mathrm{TW}_{\max }$ should be considered. Similarly for $\mathrm{V}_{\max }$. These voltages are now annotated on the cells and STA is performed. 


\subsection{Using a Comprehensive Derate factor}

In order to get more realistic performances of all the cells in the design, we have to consider more realistic Temperature and Voltage values. In traditional STA tools, the timing analysis is typically performed at 4 PVT corners, 2 for Setup check and 2 for Hold check. Therefore the standard cell library is also characterized at only these 4 PVT corners. By developing accurate derating factors we can obtain the slack values for the exact required temperature and bridge the gap between the Temperature corners used. In [4], an analytical expression is developed for the non-linear derate factor considering the supply voltage sensitivity, temperature sensitivity and dependency on complex gates

\section{NEXT-GEN TECHNIQUE: AN INTEGRATED PVT AWARE TIMING ANALYSIS}

The need for an integrated Timing analysis technique which takes into consideration the effect of all the 3 parameters (Process, Voltage and Temperature) that vary across a chip has become apparent. STA tools will have to adopt new analysis techniques that comprehensively model the impact of these variables on the design and provide more realistic results. For the designs in lower technology nodes, the STA tools in the industry currently introduce a huge pessimism and large set of paths will be marked as violating. Therefore, there is a need to remove this inherent pessimism and achieve faster design closure.



Figure 2 An integrated PVT-aware Timing Signoff Methodology

\section{CONCLUSION}

The various Timing Analysis models that are implemented in the STA tools that are currently used in the industry are discussed in detail. Their drawbacks and short-comings are discussed. The key challenge in the field of Timing Signoff in VLSI circuits now is to develop an integrated signoff methodology that considers the impact of all the on-chip variations and models them accurately. With VLSI chip designs moving towards lower technology nodes, the timing margins available are very small. The tools can no longer afford to perform timing analysis by using a guard-band based approach. The actual values of the parameters need to be considered for the analysis. The timing results cannot afford to have too much pessimism. At the same time, they have to be capable of identifying the real violations that will show up on Silicon. This need has created an opportunity for many timing industry leaders to rise to 
the occasion and develop tools that provide an integrated solution for the current problems in Timing Signoff of VLSI designs.

\section{ACKNOWLEDGMENT}

I thank my guide Dr. Kiran V, Associate Professor, Department of Electronic and Communication Engineering, for his guidance in preparing this paper. I am extremely thankful to my institute, R V College of Engineering for providing me an opportunity to write this paper. I take this opportunity to thank my family members and friends who provided all the backup support during this process.

\section{REFERENCES}

[1] D. Kouroussis, R. Ahmadi, and F. Najm, (2016) "Voltage-aware static timing analysis", IEEE Transactions on Computer-Aided Design of Integrated Circuits and Systems, vol. 25, no. 10, pp. 2156-2169.

[2] G. Bai, S. Bobba, and I. N. Hajj, (2011) "Static timing analysis including power supply noise effect on propagation delay in VLSI circuits", in Proceedings of the 38th conference on Design automation - DAC '01, ACM Press.

[3] Y.M. Jiang and K.T. Cheng, (1999) "Analysis of performance impact caused by power supply noise in deep submicron devices", in Proceedings 1999 Design Automation Conference (Cat. No. 99CH36361), IEEE.

[4] B. Lasbouygues, R. Wilson, N. Azemard, and P. Maurine, (2017) "Temperature and voltage aware timing analysis: Application to voltage drops", in 2007 Design, Automation \& Test in Europe Conference \& Exhibition, IEEE.

[5] M. Graziano, C. Forzan, and D. Pandini, (2015) "Power supply selective mapping for accurate timing analysis", in Lecture Notes in Computer Science, Springer Berlin, Heidelberg, pp. 267-276.

[6] S. Pant and D. Blaauw, (2005) "Static timing analysis considering power supply variations", in ICCAD-2005. IEEE/ACM International Conference on Computer-Aided Design, IEEE.

[7] N. V. Arvind, K. A. Rajagopal, H. S. Ajith and S. Das, (2014) "Path based approach for crosstalk delay analysis," 17th International Conference on VLSI Design. Proceedings, Mumbai, India, pp. 727-730.

[8] R. Vishweshwara, R. Venkatraman, U. H, and A. N. V, (2009) "An approach to measure the performance impact of dynamic voltage fluctuations using static timing analysis", in 2009 22nd International Conference on VLSI Design, IEEE.

[9] Y. Yamato, T. Yoneda, K. Hatayama and M. Inoue, (2012) "A fast and accurate per-cell dynamic IR-drop estimation method for at-speed scan test pattern validation," 2012 IEEE International Test Conference, Anaheim, CA, pp. 1-8. 